so to speak, in the curve of final set representing that period in the process of clinkering where we have most complete combination of the silica, but where the tricalcium silicate, characteristic of Portland cement, has not as yet formed, or, at least, separated from solic solution. The rise in the curve between $1352^{\circ} \mathrm{C}$. and $1475^{\circ} \mathrm{C}$. is not improbably due to the gradual separation or formation of tricalcium silicate, perhaps accompanied by progressive dissociation of dicalcium aluminate and ferrite. $I 475^{\circ} \mathrm{C}$. is that temperature at which the first perfect boiling test is obtained. Whether the gradual falling off in the time of final set with rise of temperature above $1475^{\circ} \mathrm{C}$. is due to dissociation of tricalcium silicate and formation of the more quick-setting dicalcium silicate we are not prepared at this time to venture an opinion, since our work has not as yet reached a point which would justify a positive opinion in regard to the behavior of the silicates themselves.

Experiment I Io would have been much more satisfactory had the collection of samples begun at as low a temperature as was employed

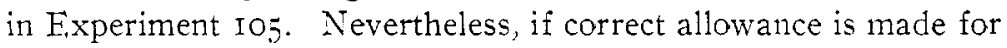
the first few samples of clinker probably not being quite as hot as is indicated by the thermocouple, we find that the curves are of very much the same nature and indicate that probably the same reactions took place in both cases. In forming this judgment due allowance must be made for the fact that in Experiment I to the individual pieces of clinker weighed twice as much as those in Experiment 105 , and that the furnace lining during the collection of the first two or three samples had probably not had time to approach the temperature of the thermocouple as in Experiment 105.

AIN ARBOR, MICHIGAN,

June 25, I9C4.

CONTRIBUTIONS FROM THE CHEMICAL LABORATORY OF THE UNIVERSITY of CrNCINNATI, No 62.]

\title{
MAGNESIUM AMALGAM AS A REDUCING AGENT.
}

By 'Thomas Evans and William C. Fetsch,

Received Iuly 9, I904.

WHILE magnesium amalgam has been known for some time, having been prepared by Wanklyn and Chapman in I866, its use as a reducing agent in organic chemistry has hitherto been neglected. 
Wanklyn and Chapman made the amalgam by heating mercury to a point a little below boiling and introducing magnesium ribbon. They state that a I per cent. amalgam so prepared decomposes water more rapidly than a I per cent. sodium amalgam, but give no data in substantiation of this.

Klauer prepared magnesium amalgam by the action of potassium amalgam upon crystalline magnesium sulphate, but did not investigate the product further than to submit it to distillation, when the mercury distilled off, leaving the magnesium in the retort.

Some years ago one of us found that magnesium powder and mercury would amalgamate at the ordinary laboratory temperature; the amalgamation starts slowly, evolving heat, which causes the process to proceed more rapidly.

Later it was found that some samples of magnesium from reputable manufacturers could not be made to amalgamate in the cold. A satisfactory method of procedure was found to consist in warming a porcelain mortar by burning a few cubic centimeters of alcohol in it, adding the mercury and rubbing in the magnesium, a little at a time. The magnesium dissolves in the mercury and separates out as a mass of beautiful silvery crystals, which soon become dull on exposure to the air.

The first amalgam prepared by the writers was nuade from I part magnesium and 9 parts mercury, and was afterwards proven to contain unamalgamated magnesium. A more homogeneous product was obtained by using I part magnesium to 18 parts mercury, practically $\mathrm{MgHg}_{2}$.

This product is thickly fluid when first prepared and sets to a hard crystalline mass on cooling.

Both the Io per cent. and 5 per cent. amalgams decomposed water rapidly with evolution of hydrogen, but were without apparent action upon cold alcahol which had been rendered absolute by distilling with sodium. Upon long-continued boiling of absolute alcohol with the amalgam, hydrogen was evolved and a slightly yellowish solution was obtained, which, upon filtration, evaporation and ignition left a residue of magnesia, thus indicating the formation of magnesium ethylate, or that a certain amount of the hydroxide dissolved in the alcohol. The quantity of the solid product 
obtained was so small that a more thorough investigation was not attempted.

The fact that the amalgam is without action upon absolute alcohol in the cold, but readily reacts upon alcolol containing a trace of water, makes it convenient for the preparation and preservation of absolute alcohol.

\section{REDUCTION OF NITROBENZENE.}

The variety of products obtainable by reducing nitrobenzene in acid, neutral or alkaline solutions, made the action of magnesium amalgam a question of interest.

Ten grams nitrobenzene were clissolved in $5^{\circ} \mathrm{cc}$. ethyl alcohol in a flask connected with an upright condenser and 40 grams of a Io per cent. magnesium amalgam added in two portions, of 20 grams each. This was a little more than the amount theoretically required to reduce the nitrobenzene to phenylhydroxylamine. The reduction started as soon as the amalgam was added, a brisk evo111tion of hyclogen taking place; the temperature rose rapidly and the solvent boiled; the flasl: was then immersed in the tap water at a temperature of $I 8^{\circ}$, moderating the reaction. Throughout the rest of the reaction the temperature was held slightly below the boiling-point of the alcohol. When no further evolution of hydrogen was apparent the second portion of the amalgam was added. When the evolution of hydrogen had ceased no odor of nitrobenzene was perceptible. The solution was filtered by means of suction from the insoluble magnesium lyydroxide and mercury, and washed with boiling methyl alcohol. Upon concentrating the reddish yellow filtrate and washings and allowing the resultant liquid to cool, characteristic plates of azobenzene crystallized out. After recrystallization these melted at $68^{\circ}$. the melting-point of azobenzene.

Having ascertained the nature of the chief product of the reduction, the experiment was repeated in order to ascertain how much of the nitrobenzene was reduced to azobenzene.

I 5.26 grams nitrobenzene gave 10.8 grams azobenzene, a yield of 95.66 per cent. of the theory. Some of the remaining, 4.3 per cent., can be accounted for by the difficulty experienced in freeing 
the precipitated magnesium hydroxide and mercury from azobenzene. Even after repeated extraction by boiling with methyl alcohol the precipitate showed red crystals when dried and examined with a magnifying glass.

No evidence of the production of hydrazobenzene was obtained, and it was at first supposed that this was due to the use of practically the theoretical amount of the reducing agent, since in other respects the magnesium amalgam resembled sodium amalgam in its action. Additional work with larger amounts of the amalgam, however, showed that even then no hydrazobenzene was formed.

[Contribution from the Chemical Laboratory of the University of Cincinnati, No. 63.]

\section{THE REDUCING ACTION OF MAGNESIUM AMALGAM UPON AROMATIC NITRO COMPOUNDS.}

BY THOMAS EVANS AND HARRY S. FRY.

Received July 9, I904.

Fvins and Fetsch ${ }^{1}$ reduced nitrobenzene with magnesium amalgam, and as some of the reductions attempted by them with ethyl alcohol as a solvent gave unsatisfactory results, it was deemed advisable to investigate the reduction of nitrobenzene in both ethyl and methyl alcohol.

Finding that the nature of the products of the reduction were dependent upon the nature of the solvent, the concentration and time, a more extencled study of the subject was made.

Nitrobenzene in Ethyl Alcohol.-Twenty-five grams of nitrobenzene were dissolved in $\mathrm{I} / 4$ liters of 93 per cent. ethyl alcohol at $15^{\circ}$ in a glass cylinder $10 \mathrm{~cm}$. in diameter, and $30 \mathrm{~cm}$. high, provided with a mechanical agitator. An amount of coarsely powdered magnesium amalgam, theoretically sufficient to convert the nitrobenezene into aniline, was then added in small portions to the violently agitated solution. At each addition there was a rapid evolution of hydrogen, accompanied by a precipitation of magnesium hydroxide and mercury in a fine state of division. The solution darkened slightly and the temperature rose from $15^{\circ}$ to $45^{\circ}$ during the reaction. When all the amalgam had been decomposed and the temperature began to fall the thinly fluid, green-

1 see preceding article. 